\title{
Signless Laplacian Polynomial and Characteristic Polynomial of a Graph
}

\author{
Harishchandra S. Ramane, ${ }^{1}$ Shaila B. Gudimani, ${ }^{2}$ and Sumedha S. Shinde ${ }^{2}$ \\ ${ }^{1}$ Department of Mathematics, Gogte Institute of Technology, Udyambag, Belgaum 590008, India \\ ${ }^{2}$ Department of Mathematics, B. V.Bhoomaraddi College of Engineering \& Technology, Hubli 580031, India
}

Correspondence should be addressed to Harishchandra S. Ramane; hsramane@yahoo.com

Received 21 July 2012; Accepted 6 September 2012

Academic Editor: Kinkar C. Das

Copyright (c) 2013 Harishchandra S. Ramane et al. This is an open access article distributed under the Creative Commons Attribution License, which permits unrestricted use, distribution, and reproduction in any medium, provided the original work is properly cited.

\begin{abstract}
The signless Laplacian polynomial of a graph $G$ is the characteristic polynomial of the matrix $Q(G)=D(G)+A(G)$, where $D(G)$ is the diagonal degree matrix and $A(G)$ is the adjacency matrix of $G$. In this paper we express the signless Laplacian polynomial in terms of the characteristic polynomial of the induced subgraphs, and, for regular graph, the signless Laplacian polynomial is expressed in terms of the derivatives of the characteristic polynomial. Using this we obtain the characteristic polynomial of line graph and subdivision graph in terms of the characteristic polynomial of induced subgraphs.
\end{abstract}

\section{Introduction}

Let $G$ be a simple graph with $n$ vertices and $m$ edges. Let the vertex set of $G$ be $V(G)=\left\{v_{1}, v_{2}, \ldots, v_{n}\right\}$. Let $\operatorname{deg}_{G}(v)$ denote the degree of a vertex $v$ in $G$. The adjacency matrix of a graph $G$ is $A(G)=\left[a_{i j}\right]$, where $a_{i j}=1$ if $v_{i}$ is adjacent to $v_{j}$ and $a_{i j}=0$, otherwise. The characteristic polynomial of a graph $G$ is defined as $\phi(G: \lambda)=\operatorname{det}(\lambda I-A(G))$, where $I$ is an identity matrix of order $n$. The degree matrix of a graph $G$ is the diagonal matrix $D(G)=\operatorname{diag}\left[d_{1}, d_{2}, \ldots, d_{n}\right]$, where $d_{i}=$ $\operatorname{deg}_{G}\left(v_{i}\right), i=1,2, \ldots, n$. The matrix $C(G)=D(G)-A(G)$, is called the Laplacian matrix and the matrix $Q(G)=D(G)+$ $A(G)$ is called the signless Laplacian matrix or Q-matrix of $G$. The characteristic polynomial of $C(G)$, defined as $C(G: \lambda)=$ $\operatorname{det}(\lambda I-C(G))$, is called the Laplacian polynomial of $G$. The characteristic polynomial of $Q(G)$, defined as $Q(G: \lambda)=$ $\operatorname{det}(\lambda I-Q(G))$, is called the signless Laplacian polynomial or Q-polynomial of a graph $G$, where $I$ is an identity matrix of order $n$.

Several results on Laplacian of $G$ are reported in the literature (see, e.g., [1-4]). Recently signless Laplacian attracted the attention of researchers [5-12]. In [13], the Laplacian polynomial of a graph is expressed in terms of the characteristic polynomial of the induced subgraphs. In this paper we express signless Laplacian polynomial of a graph in terms of the characteristic polynomial of its induced subgraphs. Further the signless Laplacian polynomial of a regular graph is expressed in terms of the derivatives of its characteristic polynomial. Using these results, we express characteristic polynomial of line graph and of subdivision graph in terms of the characteristic polynomial of its induced subgraphs.

We use standard terminology of graph theory [14].

\section{Signless Laplacian Polynomial in terms of Characteristic Polynomial}

Let the set $M_{k}(G)=\{S \mid S \subseteq V(G)$ and $|S|=k\}$, $k=0,1,2, \ldots, n$. Note that $\left|M_{k}(G)\right|=\left(\begin{array}{c}n \\ k\end{array}\right)$. We denote the product of degrees of the vertices of $G$ which belongs to $S$ by $P_{G}(S)$, that is, $P_{G}(S)=\prod_{v \in S} \operatorname{deg}_{G}(v)$. The graph $G-S$ is an induced subgraph of $G$ with vertex set $V(G)-S$. If $S=V(G)$, then $G-S=K_{0}$, a graph without vertices. Note that $\phi\left(K_{0}: \lambda\right)=1$.

Theorem 1 (see [15] ( $k$ th derivative)). Let $G$ be a graph with $n$ vertices, then

$$
\frac{d^{k}}{d \lambda^{k}} \phi(G: \lambda)=k ! \sum_{S \in M_{k}(G)} \phi(G-S: \lambda), \quad 0 \leq k \leq n
$$


Theorem 2. Let $G$ be a graph with $n$ vertices, then

$$
Q(G: \lambda)=\sum_{k=0}^{n}\left\{(-1)^{k} \sum_{S \in M_{k}(G)}\left(P_{G}(S)\right) \phi(G-S: \lambda)\right\} .
$$

Proof. Let $A(G)=\left[a_{i j}\right], 1 \leq i, j \leq n$ be the adjacency matrix of $G$ and $D(G)=\operatorname{diag}\left[d_{1}, d_{2}, \ldots, d_{n}\right]$, where $d_{i}=\operatorname{deg}_{G}\left(v_{i}\right)$, $i=1,2, \ldots, n$. Then,

$$
\begin{aligned}
Q(G: \lambda) & =\operatorname{det}(\lambda I-Q(G)) \\
& =\operatorname{det}(\lambda I-D(G)-A(G)) \\
& =\left|\begin{array}{ccccc}
\lambda-d_{1} & -a_{12} & -a_{13} & \cdots & -a_{1 n} \\
-a_{21} & \lambda-d_{2} & -a_{23} & \cdots & -a_{2 n} \\
-a_{31} & -a_{32} & \lambda-d_{3} & \cdots & -a_{3 n} \\
\vdots & & & \vdots & \\
-a_{n 1} & -a_{n 2} & -a_{n 3} & \cdots & \lambda-d_{n}
\end{array}\right| .
\end{aligned}
$$

Splitting the determinant of (3) as a sum of two determinants, we get

$$
\begin{aligned}
Q(G: \lambda)= & \left|\begin{array}{ccccc}
\lambda & -a_{12} & -a_{13} & \cdots & -a_{1 n} \\
-a_{21} & \lambda-d_{2} & -a_{23} & \cdots & -a_{2 n} \\
-a_{31} & -a_{32} & \lambda-d_{3} & \cdots & -a_{3 n} \\
\vdots & & & \vdots & \\
-a_{n 1} & -a_{n 2} & -a_{n 3} & \cdots & \lambda-d_{n}
\end{array}\right| \\
& +\left|\begin{array}{ccccc}
-d_{1} & 0 & 0 & \cdots & 0 \\
-a_{21} & \lambda-d_{2} & -a_{23} & \cdots & -a_{2 n} \\
-a_{31} & -a_{32} & \lambda-d_{3} & \cdots & -a_{3 n} \\
\vdots & & & \vdots & \\
-a_{n 1} & -a_{n 2} & -a_{n 3} & \cdots & \lambda-d_{n}
\end{array}\right| .
\end{aligned}
$$

Again splitting each of the determinants of (4) as a sum of two determinants and continuing the procedure in succession, at $n$th step we get

$$
\begin{aligned}
& Q(G) \\
& =|\lambda I-A(G)| \\
& \quad+\sum_{1 \leq i \leq n}\left(-d_{i}\right)\left|\lambda I-A\left(G-v_{i}\right)\right| \\
& \quad+\sum_{1 \leq i<j \leq n}\left(-d_{i}\right)\left(-d_{j}\right)\left|\lambda I-A\left(G-v_{i}-v_{j}\right)\right|+\cdots \\
& \quad+\sum_{1 \leq i<j<\cdots<l \leq n}\left(-d_{i}\right)\left(-d_{j}\right) \cdots\left(-d_{l}\right) \\
& \quad \times\left|\lambda I-A\left(G-v_{i}-v_{j}-\cdots-v_{l}\right)\right| .
\end{aligned}
$$

(in the above expression, $I$ stands for the identity matrix of the appropriate order and $G-v_{i}$ is a vertex deleted subgraph of $G$ ) and

$$
\begin{aligned}
Q(G & : \lambda) \\
= & |\lambda I-A(G)| \\
& +(-1) \sum_{S \in M_{1}(G)}\left(P_{G}(S)\right)|\lambda I-A(G-S)| \\
& +(-1)^{2} \sum_{S \in M_{2}(G)}\left(P_{G}(S)\right)|\lambda I-A(G-S)|+\cdots \\
& +(-1)^{n} \sum_{S \in M_{n}(G)}\left(P_{G}(S)\right)|\lambda I-A(G-S)| \\
= & \sum_{k=0}^{n}\left\{\sum _ { ( - 1 ) ^ { k } \sum _ { S \in M _ { k } ( G ) } ( P _ { G } ( S ) ) | \lambda I - A ( G - S ) | \} } ^ { n } \left\{\sum_{k=0}^{n}\left\{(-1)^{k} \sum_{S \in M_{k}(G)}\left(P_{G}(S)\right) \phi(G-S: \lambda)\right\} .\right.\right.
\end{aligned}
$$

Corollary 3. If $G$ is an r-regular graph with $n$ vertices, then

$$
Q(G: \lambda)=\sum_{k=0}^{n}(-1)^{k} \frac{r^{k}}{k !} \frac{d^{k}}{d \lambda^{k}} \phi(G: \lambda) \text {. }
$$

Proof. The graph $G$ is an $r$-regular graph, therefore $P_{G}(S)=$ $r^{k}$ for all $S \in M_{k}(G)$. Substituting this in (2) gives that

$$
\begin{aligned}
Q(G: \lambda) & =\sum_{k=0}^{n}\left\{(-1)^{k} \sum_{S \in M_{k}(G)} r^{k} \phi(G-S: \lambda)\right\} \\
& =\sum_{k=0}^{n}(-1)^{k} r^{k} \sum_{S \in M_{k}(G)} \phi(G-S: \lambda) \\
& =\sum_{k=0}^{n}(-1)^{k} \frac{r^{k}}{k !} \frac{d^{k}}{d \lambda^{k}} \phi(G: \lambda) \text { from (1). }
\end{aligned}
$$

\section{Characteristic Polynomial of Line Graph and Subdivision Graph}

The line graph $L(G)$ of a graph $G$ is the graph whose vertex set has one-to-one correspondence to the edge set of $G$ and two vertices in $L(G)$ are adjacent if and only if the corresponding edges have a vertex in common in $G$.

Theorem 4. Let $G$ be a graph with $n$ vertices and $m$ edges and $L(G)$ is the line graph of $G$, then

$$
\begin{aligned}
\phi(L(G): \lambda) \\
=(\lambda+2)^{m-n} \\
\quad \times \sum_{k=0}^{n}\left\{(-1)^{k} \sum_{S \in M_{k}(G)}\left(P_{G}(S)\right) \phi(G-S: \lambda+2)\right\} .
\end{aligned}
$$


Proof. If $G$ is a graph with $n$ vertices and $m$ edges, then [8]

$$
\phi(L(G): \lambda)=(\lambda+2)^{m-n} \mathrm{Q}(G: \lambda+2) .
$$

From (2),

$Q(G: \lambda+2)$

$$
=\sum_{k=0}^{n}\left\{(-1)^{k} \sum_{S \in M_{k}(G)}\left(P_{G}(S)\right) \phi(G-S: \lambda+2)\right\} .
$$

Substituting (11) in (10), the result follows.

Corollary 5. If $G$ is an $r$-regular graph with $n$ vertices, then

$$
\begin{aligned}
& \phi(L(G): \lambda) \\
& \quad=\left.(\lambda+2)^{n(r-2) / 2} \sum_{k=0}^{n}(-1)^{k} \frac{r^{k}}{k !} \frac{d^{k}}{d x^{k}} \phi(G: x)\right|_{x=\lambda+2} .
\end{aligned}
$$

Proof. The graph $G$ is an $r$-regular graph, therefore $P_{G}(S)=$ $r^{k}$ for all $S \in M_{k}(G)$ and the number of edges of $G$ is $m=$ $n r / 2$.

Therefore, from (9),

$$
\begin{aligned}
\phi(L(G): \lambda) \\
=(\lambda+2)^{n(r-2) / 2} \sum_{k=0}^{n}\left\{(-1)^{k} \sum_{S \in M_{k}(G)} r^{k} \phi(G-S: \lambda+2)\right\} \\
=(\lambda+2)^{n(r-2) / 2} \sum_{k=0}^{n}\left\{(-1)^{k} r^{k} \sum_{S \in M_{k}(G)} \phi(G-S: x)\right\}_{x=\lambda+2} \\
=(\lambda+2)^{n(r-2) / 2} \sum_{k=0}^{n}(-1)^{k} \frac{r^{k}}{k !} \\
\quad \times\left.\frac{d^{k}}{d x^{k}} \phi(G: x)\right|_{x=\lambda+2} \text { from (1). }
\end{aligned}
$$

The subdivision graph $S(G)$ of a graph $G$ is the graph obtained from $G$ by inserting a new vertex into every edge of $G$.

Theorem 6. Let $G$ be a graph with $n$ vertices, $m$ edges and $S(G)$ is the subdivision graph of $G$, then

$$
\begin{aligned}
& \phi(S(G): \lambda) \\
& \quad=\lambda^{m-n} \sum_{k=0}^{n}\left\{(-1)^{k} \sum_{S \in M_{k}(G)}\left(P_{G}(S)\right) \phi\left(G-S: \lambda^{2}\right)\right\} .
\end{aligned}
$$

Proof. If $G$ is a graph with $n$ vertices and $m$ edges, then [8]

$$
\phi(S(G): \lambda)=\lambda^{m-n} Q\left(G: \lambda^{2}\right) .
$$

From (2),

$$
Q\left(G: \lambda^{2}\right)=\sum_{k=0}^{n}\left\{(-1)^{k} \sum_{S \in M_{k}(G)}\left(P_{G}(S)\right) \phi\left(G-S: \lambda^{2}\right)\right\} \text {. }
$$

Substituting (16) in (15), the result follows.

Corollary 7. If $G$ is an $r$-regular graph with $n$ vertices, then

$$
\phi(S(G): \lambda)=\left.\lambda^{n(r-2) / 2} \sum_{k=0}^{n}(-1)^{k} \frac{r^{k}}{k !} \frac{d^{k}}{d x^{k}} \phi(G: x)\right|_{x=\lambda^{2}} .
$$

Proof. As $G$ is an $r$-regular graph, $P_{G}(S)=r^{k}$ for all $S \in$ $M_{k}(G)$ and the number of edges of $G$ is $m=n r / 2$.

Therefore, from (14),

$$
\begin{aligned}
& \phi(S(G): \lambda) \\
& =\lambda^{n(r-2) / 2} \sum_{k=0}^{n}\left\{(-1)^{k} \sum_{S \in M_{k}(G)} r^{k} \phi\left(G-S: \lambda^{2}\right)\right\} \\
& =\lambda^{n(r-2) / 2} \sum_{k=0}^{n}\left\{(-1)^{k} r^{k} \sum_{S \in M_{k}(G)} \phi(G-S: x)\right\}_{x=\lambda^{2}} \\
& =\left.\lambda^{n(r-2) / 2} \sum_{k=0}^{n}(-1)^{k} \frac{r^{k}}{k !} \frac{d^{k}}{d x^{k}} \phi(G: x)\right|_{x=\lambda^{2}} \text { from (1). }
\end{aligned}
$$

\section{Number of Spanning Trees}

If $G$ is an $r$-regular graph, then

$$
\begin{aligned}
Q(G: \lambda) & =|\lambda I-Q(G)|=|\lambda I-D(G)-A(G)| \\
& =|(\lambda-r) I-A(G)| \quad \text { since } D=r I \\
& =\phi(G: \lambda-r)
\end{aligned}
$$

which gives that

$$
Q(G: 2 r-\lambda)=\phi(G: r-\lambda) .
$$

Also for an $r$-regular graph $G[3,16]$,

$$
C(G: \lambda)=(-1)^{n} \phi(G: r-\lambda) .
$$
16]

Let $\tau(G)$ be the number of spanning trees of $G$, then [3,

$$
\tau(G)=\left.\frac{(-1)^{n-1}}{n} \frac{d}{d \lambda} C(G: \lambda)\right|_{\lambda=0} .
$$

Here we give the number of spanning trees using signless Laplacian.

Theorem 8. If $G$ is an $r$-regular graph with $n$ vertices, then

$$
\tau(G)=\left.\frac{1}{n} \frac{d}{d \lambda} Q(G: \lambda)\right|_{\lambda=2 r}
$$


Proof. From (22),

$$
\begin{aligned}
\tau(G) & \\
& =\left.\frac{(-1)^{n-1}}{n} \frac{d}{d \lambda} C(G: \lambda)\right|_{\lambda=0} \\
& =\left.\frac{(-1)^{n-1}}{n} \frac{d}{d \lambda}(-1)^{n} \phi(G: r-\lambda)\right|_{\lambda=0} \quad \text { from }(21), \\
& =\left.\frac{(-1)^{n-1}}{n} \frac{d}{d \lambda}(-1)^{n} Q(G: 2 r-\lambda)\right|_{\lambda=0} \quad \text { from }(20) \\
& =\left.\frac{(-1)^{n-1}}{n} \frac{d}{d \lambda}(-1)^{n}(-1) Q(G: \lambda)\right|_{\lambda=2 r} \\
& =\left.\frac{1}{n} \frac{d}{d \lambda} Q(G: \lambda)\right|_{\lambda=2 r} .
\end{aligned}
$$

\section{Conclusion}

Equation (2) establishes the relationship between the signless Laplacian polynomial and characteristic polynomial of a graph. Equations (9) and (14) express the characteristic polynomial of line graph and of subdivision graph in terms of the characteristic polynomial of a graph. As corollaries of these results, (12) and (17) give the characteristic polynomial of line graph and the subdivision graph of a regular graph in terms of the derivatives of the characteristic polynomial of a graph. These results are different from those obtained in $[17,18]$.

\section{References}

[1] R. Li, "Some lower bounds for Laplacian energy of graphs," International Journal of Contemporary Mathematical Sciences, vol. 4, no. 5, pp. 219-223, 2009.

[2] Z. Liu, "Energy, Laplacian energy and Zagreb index of line graph, middle graph and total graph," International Journal of Contemporary Mathematical Sciences, vol. 5, no. 18, pp. 895-900, 2010.

[3] B. Mohar, "The Laplacian spectrum of graphs," in Graph Theory, Combinatorics and Applications, Y. Alavi, G. Chartrand, O. E. Ollerman, and A. J. Schwenk, Eds., pp. 871-898, John Wiley \& Sons, New York, NY, USA, 1991.

[4] B. Mohar, "Graph Laplacians," in Topics in Algebraic Graph Theory, L. W. Beineke and R. J. Wilson, Eds., pp. 113-136, Cambridge University Press, Cambridge, UK, 2004.

[5] D. Cvetković, "Signless Laplacians and line graphs," Bulletin, Classe des Sciences Mathématiques et Naturelles, Sciences Mathématiques Naturelles/Sciences Mathematiques, vol. 131, no. 30, pp. 85-92, 2005.

[6] D. Cvetković, P. Rowlinson, and S. K. Simić, "Signless Laplacians of finite graphs," Linear Algebra and Its Applications, vol. 423, no. 1, pp. 155-171, 2007.

[7] D. Cvetković, P. Rowlinson, and S. K. Simić, "Eigenvalue bounds for the signless Laplacian," Publications de l'Institut Mathématique, vol. 81, no. 95, pp. 11-27, 2007.

[8] D. Cvetković, P. Rowlinson, and S. Simić, An Introduction to the Theory of Graph Spectra, Cambridge Univeristy Press, Cambridge, UK, 2010.
[9] A. Daneshgar and H. Hajiabolhassan, "Graph homomorphisms and nodal domains," Linear Algebra and Its Applications, vol. 418, no. 1, pp. 44-52, 2006.

[10] L. Feng and G. Yu, "On three conjectures involving the signless Laplacian spectral radius of graphs," Publications de l'Institut Mathematique, vol. 85, no. 99, pp. 35-38, 2009.

[11] W. H. Haemers and E. Spence, "Enumeration of cospectral graphs," European Journal of Combinatorics, vol. 25, no. 2, pp. 199-211, 2004.

[12] E. R. van Dam and W. H. Haemers, "Which graphs are determined by their spectrum?" Linear Algebra and Its Applications, vol. 373, pp. 241-272, 2003.

[13] H. B. Walikar and H. S. Ramane, "Laplacian polynomial and number of spanning trees in terms of characteristic polynomial of induced subgraphs," AKCE International Journal of Graphs and Combinatorics, vol. 5, no. 1, pp. 35-48, 2008.

[14] F. Harary, Graph Theory, Narosa Publishing House, New Delhi, India, 1998.

[15] H. S. Ramane, Some topics in spectral graph theory [Ph.D. thesis], Karnatak University, Dharwad, India, 2002.

[16] D. Cvetković, M. Doob, and H. Sachs, Spectra of Graphs, Academic Press, New York, NY, USA, 1980.

[17] H. Sachs, "Über Teiler, Faktoren und charakteristische Polynome von Graphen," Teil I. Wiss. Z. TH Ilmenau, vol. 13, pp. 405-412, 1967.

[18] D. Cvetković, "Spectra of graphs formed by some unary operations," Publications de l'Institut Mathematique, vol. 19, no. 33, pp. 37-41, 1975 . 


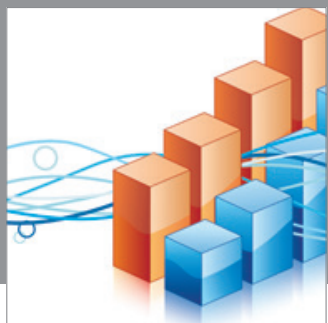

Advances in

Operations Research

mansans

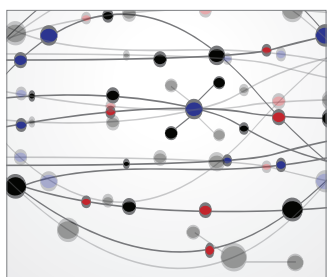

The Scientific World Journal
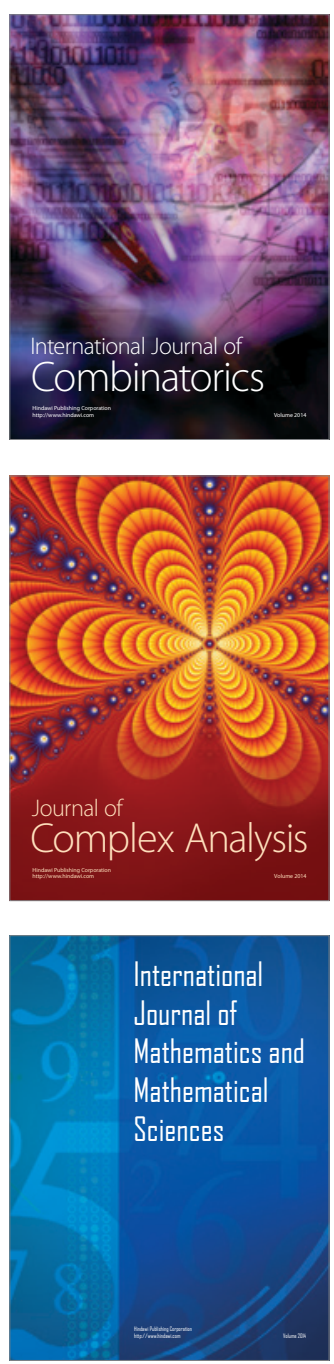
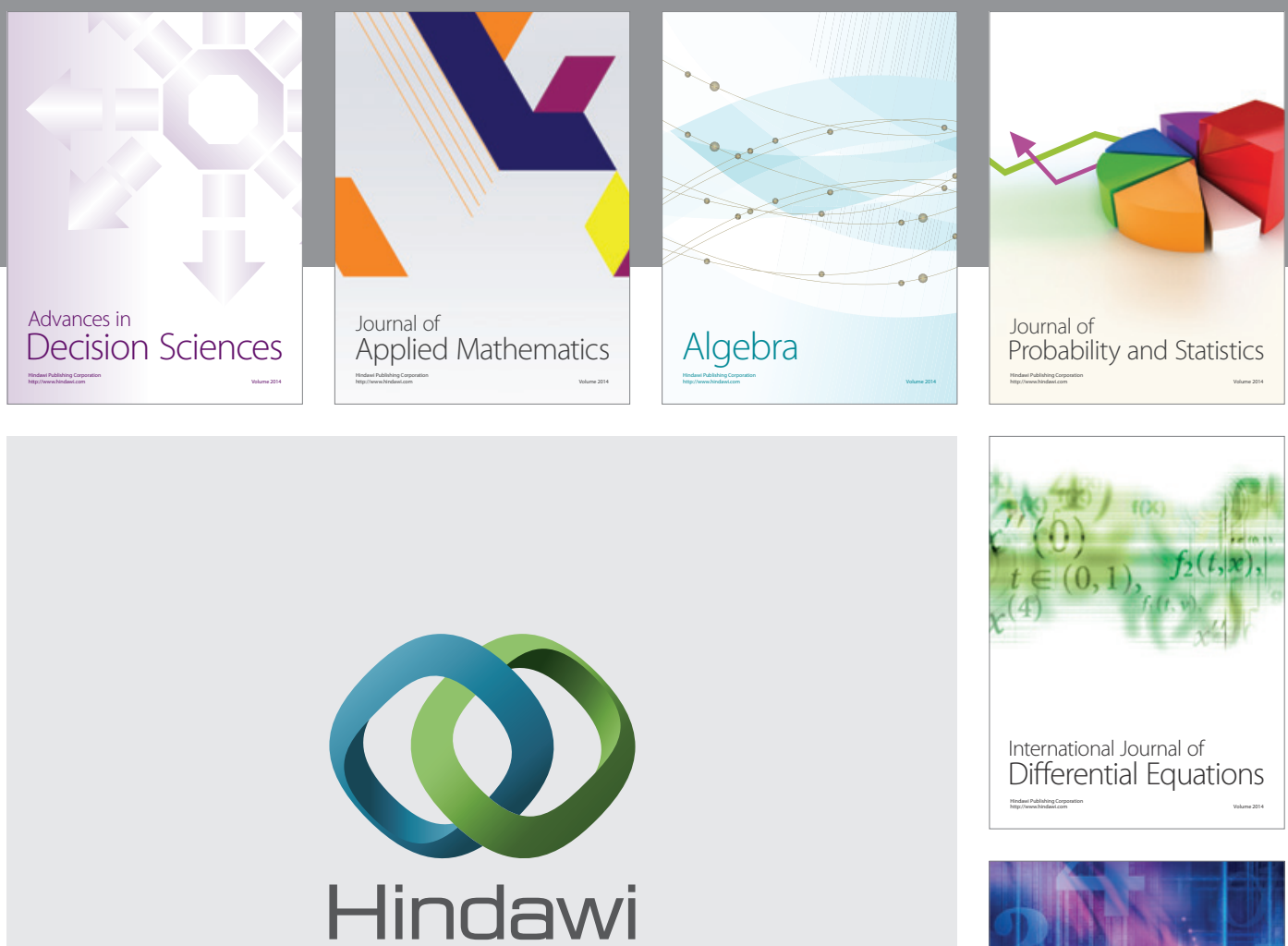

Submit your manuscripts at http://www.hindawi.com
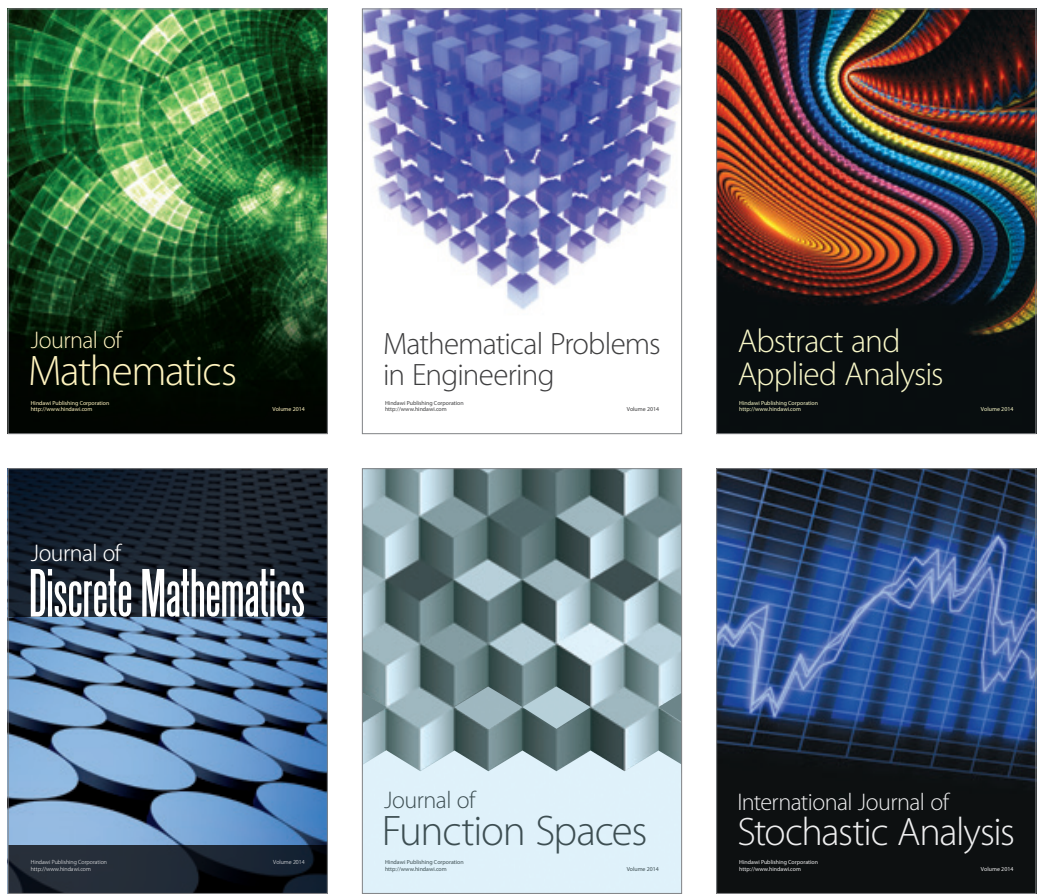

Journal of

Function Spaces

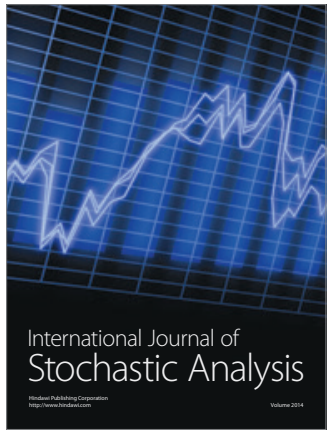

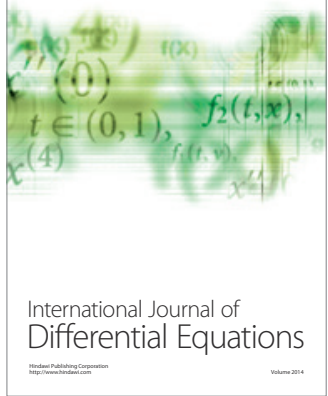
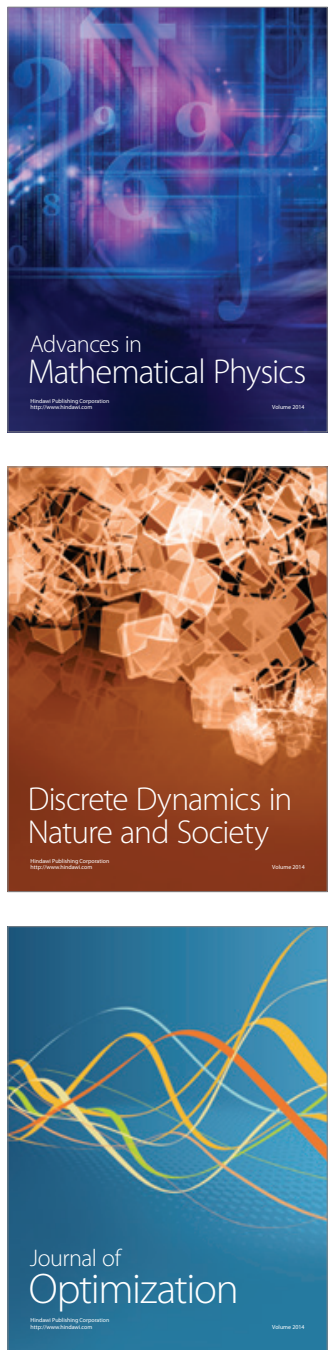